International Journal of Pure and Applied Mathematics

Volume 103 No. 4 2015, 625-633

ISSN: 1311-8080 (printed version); ISSN: 1314-3395 (on-line version)

url: http://www.ijpam.eu

doi: http://dx.doi.org/10.12732/ijpam.v103i4.3

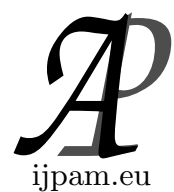

\title{
ONE MODULO THREE MEAN LABELING OF CYCLE RELATED GRAPHS
}

\author{
P. Jeyanthi ${ }^{1}$ A. Maheswari ${ }^{\S}$, P. Pandiaraj ${ }^{3}$ \\ ${ }^{1}$ Research Centre \\ Department of Mathematics \\ Govindammal Aditanar College for Women \\ Tiruchendur, 628 215, Tamilnadu, INDIA \\ ${ }^{2,3}$ Department of Mathematics \\ Kamaraj College of Engineering and Technology \\ Virudhunagar, Tamilnadu, INDIA
}

\begin{abstract}
The concept of one modulo three mean labeling was introduced in [2]. In this paper, we prove that the graphs $E J_{n}, P_{4 m}(+) \overline{K_{n}}, K_{1,2 n} \times$ $P_{2}, N A\left(Q_{m}\right), S^{\prime}\left(P_{2 n}\right), D\left(C_{n}, v^{\prime}\right)$ and $D\left(C_{n}, e^{\prime}\right)$ are one modulo three mean graphs.
\end{abstract}

AMS Subject Classification: $05 \mathrm{C} 78$

Key Words: one modulo three mean labeling, one modulo three mean graphs

\section{Introduction}

All graphs considered here are simple, finite, connected and undirected. We follow the basic notations and terminologies of graph theory as in [1]. Swaminathan and Sekar introduced the notion of one modulo three graceful labeling in [4]. Motivated by the work of these authors Jeyanthi and Maheswari

Received: March 12, 2015

(C) 2015 Academic Publications, Ltd.

$\S_{\text {Correspondence author }}$ 
[2] introduced the concept of one modulo three mean labeling. A graph $G$ is said to be one modulo three mean graph if there is an injective function $\phi$ from the vertex set of $G$ to the set $\{a / 0 \leq a \leq 3 q-2$ and either $a \equiv$ $0(\bmod 3)$ or $a \equiv 1(\bmod 3)\}$ where $q$ is the number of edges of $G$ and $\phi$ induces a bijection $\phi^{*}$ from the edge set of $G$ to $\{a / 1 \leq a \leq 3 q-2$ and either $a \equiv 1(\bmod 3)\}$ given by $\phi^{*}(u v)=\left\lceil\frac{\phi(u)+\phi(v)}{2}\right\rceil$ and the function $\phi$ is called one called one modulo three mean labeling of $G$. In [2], they proved that $P_{2 n}$, comb, bistar $B_{n, n}, T_{p}$-tree with even number of vertices, $C_{4 n+1}$, ladder $L_{n+1}, K_{1,2 n} \times K_{2}$ are one modulo three mean graphs. Also they proved that $B_{m, n}, K_{1, n}, K_{n}, n>3$ are not one modulo three mean graphs. In [3], it is proved that $D A\left(Q_{n}\right), D A\left(Q_{2}\right) \odot n K_{1}, D A\left(Q_{m}\right) \odot n K_{1}, D A\left(T_{2}\right) \odot n K_{1}, D A\left(T_{m}\right) \odot n K_{1}$, $\bar{S}\left(D A\left(T_{n}\right)\right), \bar{S}\left(D A\left(Q_{n}\right)\right), m P_{n}, m \geq 1, C_{m} *_{e} C_{n}(m, n \equiv 1(\bmod 4))$ graphs are one modulo three mean graphs. In this paper we extend our study on one modulo three mean labeling and prove that $E J_{n}, P_{4 m}(+) \bar{K}_{n}, K_{1,2 n} \times P_{2}, N A\left(Q_{m}\right)$, $S^{\prime}\left(P_{2 n}\right), D\left(C_{n}, v^{\prime}\right), D\left(C_{n}, e^{\prime}\right)$ are one modulo three mean graphs.

We use the following known theorems and definitions in the subsequent section.

Theorem 1. [4] Let $G_{1}\left(p_{1}, q_{1}\right), G_{2}\left(p_{2}, q_{2}\right), \ldots, G_{m}\left(p_{m}, q_{m}\right)$ be a one modulo three mean graphs with $q_{i}(1 \leq i \leq m)$ is odd and $u_{i}, v_{i}$ be the vertices of $G_{i}(1 \leq i \leq m)$ labeled with 0 and $3 q_{i}-2$. Then the graph $G$ obtained by joining $v_{1}$ with $u_{2}$ and $v_{2}$ with $u_{3}$ and $v_{3}$ with $u_{4}$ and so on until we join $v_{m-1}$ with $u_{m}$ by an edge is also a one modulo three mean graph.

Definition 2. Let $G$ be a graph. For each point $v$ of a graph $G$, take a new point $v^{\prime}$ and join $v^{\prime}$ to the vertices of $G$ which are adjacent to $v$. The graph thus obtained is called the splitting graph of $G$ and is denoted by $S^{\prime}(G)$.

Definition 3. Let $G$ be a graph and $v$ be any vertex of $G$. A new vertex $v^{\prime}$ is said to be duplication of $v$ if all the vertices which are adjacent to $v$ are adjacent to $v^{\prime}$. The graph obtained by duplication $v$ is denoted by $D\left(G, v^{\prime}\right)$.

Definition 4. Let $G$ be a graph and $e$ be any edge of $G$. A new edge $e^{\prime}$ is said to be duplication of an edge eif all the edges which are incident to $e$ in $G$ are incident to $e^{\prime}$. The graph obtained by duplication $e$ is denoted by $D\left(G, e^{\prime}\right)$.

Definition 5. An $n^{\text {th }}$ alternate quadrilateral snake $N A\left(Q_{m}\right)$ consists of $n$ alternate quadrilateral snakes that have a common path. That is, a $n^{\text {th }}$ alternate quadrilateral snake is obtained from a path $u_{1}, u_{2}, \ldots, u_{m}$ by joining $u_{2 i-1}$ and $u_{2 i}\left(1 \leq i \leq \frac{m}{2}\right)$ to the $n$ new vertices $v_{i j}$ and $w_{i j}$ respectively and then joining $v_{i j}, w_{i j}\left(1 \leq i \leq \frac{m}{2}, 1 \leq j \leq n\right)$. 
Definition 6. A cartesian product of two graphs $G_{1}$ and $G_{2}$ is the graph $G_{1} \times G_{2}$ such that its vertex set is a cartesian product of $V\left(G_{1}\right)$ and $V\left(G_{2}\right)$. That is $V\left(G_{1} \times G_{2}\right)=V\left(G_{1}\right) \times V\left(G_{2}\right)=\left\{(x, y) / x \in V\left(G_{1}\right), y \in V\left(G_{2}\right)\right\}$ and its edge set is defined as $E\left(G_{1} \times G_{2}\right)=\left\{\left(\left(x_{1}, x_{2}\right),\left(y_{1}, y_{2}\right)\right) / x_{1}=y_{1}\right.$ and $\left(x_{2}, y_{2}\right) \in E\left(G_{2}\right)$ or $x_{2}=y_{2}$ and $\left.\left(x_{1}, y_{1}\right) \in E\left(G_{1}\right)\right\}$.

Definition 7. A triangular ladder $T L_{n}, n \geq 2$ is a graph obtained from $L_{n}$ by adding the edges $u_{i} v_{i+1}, 1 \leq i \leq n-1$ where $u_{i}$ and $v_{i}$ are the vertices of $L_{n}$ such that $u_{1}, u_{2}, \ldots, u_{n}$ and $v_{1}, v_{2}, \ldots, v_{n}$ are two paths of length $n$ in the graph $L_{n}$.

Definition 8. A friendship graph $F_{n}$ is a one point union of $n$ copies of cycle $C_{3}$.

Definition 9. The extend jewel graph $E J_{n}$ is a graph with vertex set $V\left(E J_{n}\right)=\left\{u, v, x, y, w, z, u_{i}: 1 \leq i \leq n\right\}$ and edge set

$$
E\left(E J_{n}\right)=\left\{u v, u x, x y, y z, v w, w z, v u_{i}, z u_{i}: 1 \leq i \leq n\right\} .
$$

Definition 10. The composition of two graphs $G_{1}=\left(V_{1}, E_{1}\right)$ and $G_{2}=$ $\left(V_{2}, E_{2}\right)$ is a graph $G_{1}\left[G_{2}\right]$ with a vertex set $V=V_{1} \times V_{2}$ and an edge set $E=\left\{u v \mid u=\left(u_{1}, u_{2}\right), v=\left(v_{1}, v_{2}\right)\right.$ and either $u_{1} v_{1} \in E_{1}$ or $u_{1}=v_{1}$ and $\left.u_{2} v_{2} \in E_{2}\right\}$.

Definition 11. Let $G$ be a graph with two or more vertices. A total graph $T(G)$ is the graph whose vertex set is $V(G) \cup E(G)$ and the two vertices are adjacent in $T(G)$ whenever they are either adjacent or incident in $G$.

\section{Main Results}

Theorem 12. If $G$ is a graph in which every edge is an edge of a triangle, then $G$ is not a one modulo three mean graph.

Proof. Let $G$ be a graph in which every edge is an edge of a triangle. Suppose $G$ is a one modulo three mean graph. To get 1 on edge label, there must be two adjacent vertices $u$ and $v$ such that $f(u)=0$ and $f(v)=1$. Let $u v w u$ be a triangle in which the edge $u v$ lies. To get 4 on edge label, there must be $f(w)=7$, then $u w$ and $v w$ get the same edge label. This is a contradiction to the fact of one modulo three mean labeling. Hence $G$ is not a one modulo three mean labeling graph. 
Corollary 13. The wheel graph $W_{n}$, flower graph $F L_{n}$, triangular snakes, double triangular snakes, triangular ladders, fans $P_{n}+K_{1}, n \geq 2$, Double fans $P_{n}+K_{2}, n \geq 2$, friendship graph $C_{3}^{n}$, windmills $K_{m}^{n}, m>3$, square graph $B_{n, n}^{2}$, total graph $T\left(P_{n}\right)$ and composition graph $P_{n}\left[P_{2}\right]$ are not one modulo three mean graph.

Theorem 14. The extend jewel graph $E J_{n}$ is a one modulo three mean graph.

Proof. Let vertex set $V\left(E J_{n}\right)=\left\{u, v, x, y, w, z, u_{i}: 1 \leq i \leq n\right\}$ and edge set $E\left(E J_{n}\right)=\left\{u v, u x, x y, y z, v w, w z, v u_{i}, z u_{i}, x w: 1 \leq i \leq n\right\}$. Then $E J_{n}$ has $n+6$ vertices and $2 n+7$ edges. Define a vertex labeling $\phi: V(G) \rightarrow$ $\{0,1,3, \ldots, 6 n+19\}$ as follows. $f\left(u_{i}\right)=6 i+7$ if $1 \leq i \leq n, f(u)=1, f(v)=$ $0, f(w)=7, f(x)=6 n+13, f(y)=6 n+18, f(z)=6 n+19$. It can be verified that the induced edge labels of $E J_{n}$ are $1,4, \ldots, 6 n+19$. Hence $\phi$ is a one modulo three mean labeling of $E J_{n}$.

An example for the one modulo three mean labeling of $E J_{4}$ is given in Figure 1.

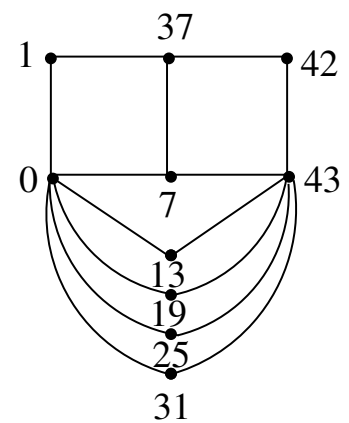

Figure 1

Theorem 15. Let $G=P_{m}(+) \bar{K}_{n}$ be the graph with the vertex set $V(G)=$ $\left\{u_{i}, v_{j}: 1 \leq i \leq m, 1 \leq j \leq n\right\}$ and the edge set $E(G)=\left\{u_{i} u_{i+1}, u_{1} v_{j}, u_{m} v_{j}\right.$ : $1 \leq i \leq m-1,1 \leq j \leq n\}$. Then $G$ is a one modulo three mean graph if $m \equiv 0(\bmod 4)$.

Proof. Let vertex set $V(G)=\left\{u_{i}, v_{j}: 1 \leq i \leq m, 1 \leq j \leq n\right\}$ and the edge set $E(G)=\left\{u_{i} u_{i+1}, u_{1} v_{j}, u_{m} v_{j}: 1 \leq i \leq m-1,1 \leq j \leq n\right\}$. Here $|V(G)|=m+n,|E(G)|=m+2 n-1$. Define a vertex labeling $\phi: V(G) \rightarrow$ $\{0,1,3, \ldots, 3 m+6 n-5\}$ as follows. $f\left(v_{n-i+1}\right)=6(n-i)+3 m+1$ if $1 \leq i \leq n$,

$$
f\left(u_{2 i-1}\right)= \begin{cases}6(i-1) & \text { if } 1 \leq i \leq \frac{m}{4} \\ 6 i-5 & \text { if } \frac{m}{4}+1 \leq i \leq \frac{m}{2},\end{cases}
$$




$$
f\left(u_{2 i}\right)= \begin{cases}6 i-5 & \text { if } 1 \leq i \leq \frac{m}{4} \\ 6(i-1)+6 n & \text { if } \frac{m}{4}+1 \leq i \leq \frac{m}{2}\end{cases}
$$

It can be verified that the induced edge labels of $G$ are $1,4, \ldots, 3 m+6 n-5$. Hence $\phi$ is a one modulo three mean labeling of $P_{m}(+) \overline{K_{n}}$. Hence $P_{m}(+) \overline{K_{n}}$ is a one modulo three mean graph if $m \equiv 0(\bmod 4)$.

An example for the one modulo three mean labeling of $P_{8}(+) \bar{K}_{3}$ is shown in Figure 2.

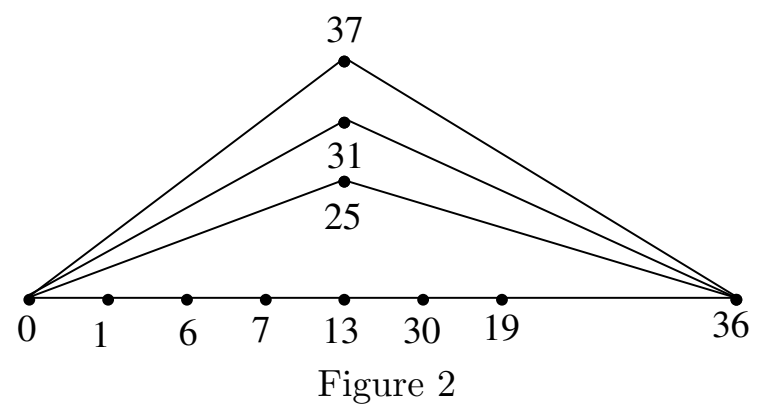

Remark 16. [4] The graph $D A\left(Q_{n}\right)$ is a one modulo three mean graph.

Theorem 17. The book graph $K_{1, n} \times P_{2}(n>2)$ is a one modulo three mean graph if and only if $n$ is even.

Proof. Let $G=K_{1, n} \times P_{2}$. The vertex set $V(G)=\left\{u, v, u_{i}, v_{i}: 1 \leq i \leq n\right\}$ and the edge set $E(G)=\left\{u v, u u_{i}, v v_{i}, u_{i} v_{i}: 1 \leq i \leq n\right\}$. Here $|V(G)|=$ $2 n+2,|E(G)|=3 n+1$. Define a vertex labeling $\phi: V(G) \rightarrow\{0,1,3, \ldots, 9 n+1\}$ as follows. $f(u)=0, f(v)=9 n+1, f\left(u_{i}\right)=6 i-5$ if $1 \leq i \leq n$.

$$
\begin{aligned}
& f\left(v_{\frac{n}{2}-i+1}\right)= \begin{cases}9 n-6 i+6 & \text { if } 1 \leq i \leq\left\lfloor\frac{n}{4}\right\rfloor \\
9 n-6 i+1 & \text { if }\left\lfloor\frac{n}{4}\right\rfloor+1 \leq i \leq \frac{n}{2}\end{cases} \\
& f\left(v_{n-i+1}\right)=6 n+6\left\lfloor\frac{n-1}{4}\right\rfloor+7 \text { if } i=\left\lfloor\frac{n-1}{4}\right\rfloor+1, \\
& f\left(v_{3}\right)=18 \text { if } n=4 . \\
& \text { If } n>4, f\left(v_{n-i+1}\right)= \begin{cases}6 n-6 i & \text { if } 1 \leq i \leq\left\lfloor\frac{n-1}{4}\right\rfloor \\
6 n-6 i+6 & \text { if }\left\lfloor\frac{n-1}{4}\right\rfloor+2 \leq i \leq \frac{n}{2}\end{cases}
\end{aligned}
$$

It can be verified that the induced edge labels of $G$ are $1,4, \ldots, 9 n+1$. Hence $\phi$ is a one modulo three mean labeling of $K_{1, n} \times P_{2}$. Hence $K_{1, n} \times P_{2}$ is a one modulo three mean graph if $n$ is even. Conversely, assume that $n \equiv 1(\bmod 2)$ and take $n=2 k+1$. Then $|V(G)|=4 k+4$ and $|E(G)|=6 k+4$. Let $\phi$ be a one modulo three mean labeling of $K_{1, n} \times P_{2}$, then $0,1,18 k+10$ and $18 k+9$ must be the vertex labels of one modulo three mean graphs. If $f(u)=0, f(v)=$ $1, f\left(u_{i}\right)=18 k+10, f\left(v_{i}\right)=18 k+9(1 \leq i \leq n)$, then the induced edge label of $u u_{i}$ and $v v_{i}$ get the same label $9 k+5$ which is not possible. Also this is a 
contradiction to the fact that the edge labels are congruent to one modulo three. If $f(u)=0, f\left(v_{i}\right)=1, f(v)=18 k+9$ (or) $f(u)=1, f\left(v_{i}\right)=0, f(v)=18 k+10$ (or) $f(v)=0, f\left(u_{i}\right)=1, f(u)=18 k+9$ (or) $f(u)=18 k+10, f(v)=1, f\left(u_{i}\right)=$ $0(1 \leq i \leq n)$, then the induced edge label is $9 k+5$ which is not possible. This is a contradiction to the fact that the edge labels are congruent to one modulo three. Therefore, 0 and $18 k+10$ cannot be the labels of the adjacent vertices. Hence the book graph $K_{1, n} \times P_{2}$ is not a one modulo three mean graph if $n$ is odd.

Theorem 18. The $n^{\text {th }}$ alternate quadrilateral snake $N A\left(Q_{m}\right)$ is a one modulo three mean graph if $n$ is even.

Proof. By Theorem 17, NA(Q $\left.Q_{2}\right)$ is a one modulo three mean graph. Let $G_{i}=N A\left(Q_{2}\right)$ for $1 \leq i \leq m-1$. Since each $G_{i}$ has $3 n+1$ edges, by Theorem 1 , $N A\left(Q_{m}\right)$ admits one modulo three mean labeling.

An example for the one modulo three mean labeling of $4 A\left(Q_{4}\right)$ is shown in Figure 3.

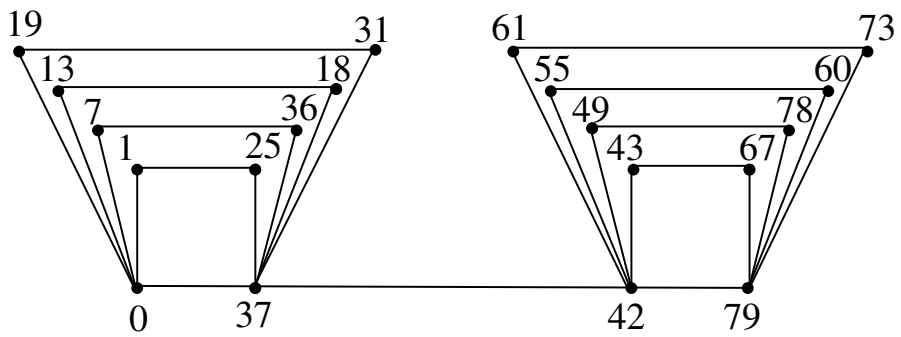

Figure 3.

Theorem 19. The splitting graph $S^{\prime}\left(P_{n}\right)$ is a one modulo three mean graph if $n$ is even.

Proof. Let $v_{1}, v_{2}, \ldots, v_{n}$ be the vertices of $P_{n}$ and $v_{1}, v_{2}, \ldots, v_{n}, v_{1}^{\prime}, v_{2}^{\prime}, \ldots, v_{n}^{\prime}$ be the vertices of $S^{\prime}\left(P_{n}\right)$. Here $S^{\prime}\left(P_{n}\right)$ has $2 n$ vertices and $3(n-1)$ edges. Define a vertex labeling $\phi: V\left(S^{\prime}\left(P_{n}\right)\right) \rightarrow\{0,1,3, \ldots, 9 n-11\}$ as follows.

$$
\begin{aligned}
& f\left(v_{i}\right)=\left\{\begin{array}{ll}
9(i-1) & \text { if } i \text { is odd } \\
9 i-11 & \text { if } i \text { is even }
\end{array},\right. \\
& f\left(v_{i}^{\prime}\right)= \begin{cases}9 i-3 & \text { if } i \text { is odd } \\
9 i-17 & \text { if } i \text { is even. }\end{cases}
\end{aligned}
$$

It can be verified that the induced edge labels of $S^{\prime}\left(P_{n}\right)$ are $1,4, \ldots, 9 n-11$. Clearly $\phi$ is a one modulo three mean labeling of $S^{\prime}\left(P_{n}\right)$. Hence $S^{\prime}\left(P_{n}\right)$ is a one modulo three mean graph if $n$ is even. 
An example for the one modulo three mean labeling of splitting graph of a path $S^{\prime}\left(P_{6}\right)$ is shown in Figure 4.

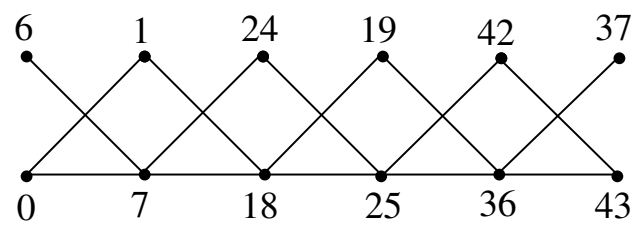

Figure 4.

Theorem 20. The graph obtained by duplicating an arbitrary vertex of a cycle $D\left(C_{n}, v^{\prime}\right)$ admits one modulo three mean labeling if $n \equiv 1,3(\bmod 4)$.

Proof. Let $v_{1}, v_{2}, \ldots, v_{n}$ be the vertices of cycle $C_{n}$. Let $G=D\left(C_{n}, v^{\prime}\right)$ be the graph obtained by duplicating an arbitrary vertex $v$ of $C_{n}$. Without loss of generality take $v=v_{n}$ and the duplication of $v_{n}$ be $v_{n}^{\prime}$. Hence $\left|V\left(D\left(C_{n}, v_{n}^{\prime}\right)\right)\right|=$ $n+1$ and $\left|E\left(D\left(C_{n}, v_{n}^{\prime}\right)\right)\right|=n+2$. Define a vertex labeling $\phi: V(G) \rightarrow$ $\{0,1,3, \ldots, 3 n+4\}$ by considering the following two cases.

Case $(\mathbf{i}) . \quad n \equiv 1(\bmod 4)$.

$$
\begin{aligned}
& f\left(v_{1}\right)=1, f\left(v_{n}\right)=13, f\left(v_{n}^{\prime}\right)=7 \text { and } \\
& f\left(v_{n-2 i+1}\right)= \begin{cases}3 n-6 i+9 & \text { if } 1 \leq i \leq\left\lfloor\frac{n}{4}\right\rfloor \\
3 n-6 i-3 & \text { if }\left\lceil\frac{n}{4}\right\rceil \leq i \leq\left\lfloor\frac{n}{2}\right\rfloor,\end{cases} \\
& f\left(v_{n-2 i}\right)=3 n-6 i+10 \text { if } 1 \leq i \leq\left\lfloor\frac{n}{2}\right\rfloor-1 .
\end{aligned}
$$

Case (ii). $n \equiv 3(\bmod 4)$.

$$
\begin{aligned}
& f\left(v_{1}\right)=3 n+4, f\left(v_{n}^{\prime}\right)=3 n+3, \\
& f\left(v_{2 i}\right)= \begin{cases}6(i-1) & \text { if } 1 \leq i \leq\left\lceil\frac{n}{4}\right\rceil \\
6 i-5 & \text { if }\left\lceil\frac{n}{4}\right\rceil+1 \leq i \leq\left\lfloor\frac{n}{2}\right\rfloor,\end{cases} \\
& f\left(v_{2 i+1}\right)= \begin{cases}6 i-5 & \text { if } 1 \leq i \leq\left\lceil\frac{n}{4}\right\rceil \\
6 i & \text { if }\left\lceil\frac{n}{4}\right\rceil+1 \leq i \leq\left\lfloor\frac{n}{2}\right\rfloor .\end{cases}
\end{aligned}
$$

It can be verified that the induced edge labels of $D\left(C_{n}, v^{\prime}\right)$ are $1,4, \ldots, 3 n+$ 4. Clearly $\phi$ is a one modulo three mean labeling of $D\left(C_{n}, v^{\prime}\right)$. Hence $D\left(C_{n}, v^{\prime}\right)$ is one modulo three mean graph if $n \equiv 1,3(\bmod 4)$.

An example for the one modulo three mean labeling of duplicating an arbitrary vertex of a cycle $D\left(C_{11}, v^{\prime}\right)$ is shown in Figure 5 . 


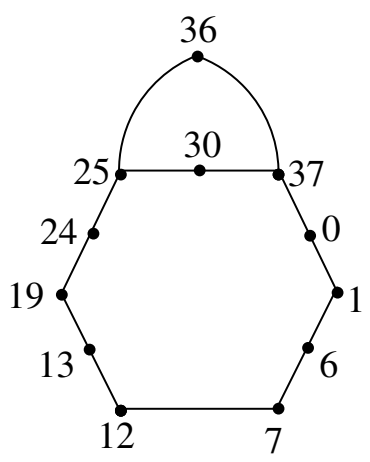

Figure 5.

Theorem 21. The graph obtained by duplicating an arbitrary edge in cycle $D\left(C_{n}, e^{\prime}\right)$ is a one modulo three mean graph if $n \equiv 0,2(\bmod 4)$.

Proof. Let $v_{1}, v_{2}, \ldots, v_{n}$ be the vertices of the cycle $C_{n}$. Let $G=D\left(C_{n}, e^{\prime}\right)$ be a graph obtained by duplicating an arbitrary edge $e$ of $C_{n}$. Without loss of generality take $e=v_{1} v_{2}$ and the duplication of $e$ be edge is $e^{\prime}=v_{1}^{\prime} v_{2}^{\prime}$. Hence $\left|V\left(D\left(C_{n}, e^{\prime}\right)\right)\right|=n+2$ and $\left|E\left(D\left(C_{n}, e^{\prime}\right)\right)\right|=n+3$. Define a vertex labeling $\phi: V(G) \rightarrow\{0,1,3,4, \ldots, 3 n+7\}$ by considering the following two cases.

Case (i). $n \equiv 2(\bmod 4)$.

$$
\begin{aligned}
& f\left(v_{1}\right)=\left\{\begin{array}{ll}
12 & \text { if } n=6 \\
19 & \text { if } n>6,
\end{array} \quad f\left(v_{2}\right)=7, f\left(v_{1}^{\prime}\right)=3 n+6, f\left(v_{2}^{\prime}\right)=1 .\right. \\
& f\left(v_{2 i+1}\right)=6(i-1) \text { if } 1 \leq i \leq\left\lfloor\frac{n}{4}\right\rfloor, f\left(v_{\frac{n}{2}+2}\right)= \begin{cases}3 n & \text { if } n=6 \\
\frac{3 n}{2}+3 & \text { if } n>6,\end{cases} \\
& f\left(v_{2 i+1}\right)=6 i+6 \text { if }\left\lfloor\frac{n}{4}\right\rfloor+2 \leq i \leq\left\lfloor\frac{n}{2}\right\rfloor-1, \\
& f\left(v_{n-2 i+2}\right)= \begin{cases}3 n-6 i+13 & \text { if } 1 \leq i \leq \frac{n}{2}-2 \\
13 & \text { if } i=\frac{n}{2}-1\end{cases}
\end{aligned}
$$

Case (ii). $n \equiv 0(\bmod 4)$.

$$
\begin{aligned}
& f\left(v_{1}\right)=12, f\left(v_{2}\right)=1, f\left(v_{1}^{\prime}\right)=3 n+6, f\left(v_{2}^{\prime}\right)=7, \\
& f\left(v_{2 i+1}\right)= \begin{cases}6(i-1) & \text { if } 1 \leq i \leq \frac{n}{4} \\
6 i+6 & \text { if } \frac{n}{4}+1 \leq i \leq \frac{n}{2}-1\end{cases}
\end{aligned}
$$

$f\left(v_{n-2 i+2}\right)=3 n-6 i+13$ if $1 \leq i \leq \frac{n}{2}-1$. It can be verified that the induced edge labels of $D\left(C_{n}, e^{\prime}\right)$ are $1,4, \ldots, 3 n+4$. Hence $\phi$ is a one modulo three mean labeling of $D\left(C_{n}, e^{\prime}\right)$. Hence $D\left(C_{n}, e^{\prime}\right)$ is a one modulo three mean graph if $n \equiv 0,2(\bmod 4)$.

An example for the one modulo three mean labeling of duplicating an arbitrary edge of a cycle $D\left(C_{10}, e^{\prime}\right)$ is shown in Figure 6. 


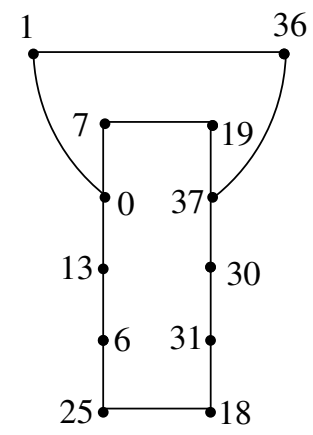

Figure 6 .

\section{References}

[1] F. Harary, Graph Theory, Addison Wesley, Massachusetts, 1972.

[2] P. Jeyanthi and A. Maheswari, One modulo three mean labeling of graphs, American Journal of Applied Mathematics and Statistics, 2(5) (2014), 302306. DOI:10.12691/ajams-2-5-2

[3] P. Jeyanthi, A. Maheswari and P. Pandiaraj, On one modulo three mean labeling of graphs, (Preprint).

[4] V. Swaminathan and C. Sekar, Modulo three graceful graphs, Proceed. National Conference on Mathematical and Computational Models, PSG College of Technology, Coimbatore, (2001), 281-286. 
